\title{
Some relations between affect and recall: An exploratory study
}

EDWARD E. SMITH AND WALTER SLOBODA, BEHA VIORAL AND CLINICAL STUDIES RESEARCH CENTER, SAINT ELIZABETHS HOSPITAL, Washington, D. C. 20032

This study investigated (1) the relation between GSRs occasioned by external events and by recall of these events, and (2) clustering in free recall based on categories of subjective pleasantness, unpleasantness, and neutrality. A set of 15 pictures (of which five were categorized as pleasant, five as unpleasant and five as neutral) were presented to $S$. When $S$ later recalled information about these pictures, there was no consistent relation between presentation and recall GSRs. In another part of the experiment $S$ attempted to recall names which had previously been given to each picture. Clustering of these names by the pleasantness, unpleasantness or neutrality of their referents (the pictures) was observed, and was attributed, in part, to S's naming subjectively similar experiences with associatively related names.

Although hundreds of experiments have been performed on the interaction of affective factors (as measured by subjective expressions and physiological responses) and recall, two important questions have been neglected: (1) What is the relation between the affect (as measured by physiological responses) occasioned by the perception of an external event and the affect occasioned by a recollection of that event?, and (2) Do affective factors influence the organization of recall? With regard to (1), the specific question at issue was: If the perception of event $A$ was associated with a greater physiological response (i.e., a greater GSR) than that of event $B$, would the recall of event $A$ be associated with a greater physiological response than the recall of event $B$, assuming the two events are equally memorable? With regard to (2), Bousfield (1953) has shown that in a free recall situation Ss organize their responses by recalling items together which belong to the same semantic category, e.g., animals or vegetables. This phenomenon is referred to as category clustering. The issue investigated in this experiment was whether categories of subjective pleasantness, unpleasantness, and neutrality could serve as bases for organizing (clustering) free recall.

Method

The 12 Ss ( 7 males and 5 females) were students attending universities in the Washington, D. C. area, and were paid for their participation.

The experiment consisted of four parts. In Part I, $S$ was shown a series of 15 pictures, each of which was exposed for $5 \mathrm{sec}$, with a $20 \mathrm{sec}$ blank interval separating the offset of one picture from the onset of the next. The $S$ was instructed to look at each picture, and to give a one or two word name for it as soon as its exposure terminated. In Part II, E read aloud each of these names and S attempted to recall the number of people present in the picture named. The $S$ was given $20 \mathrm{sec}$ in which to make his decision, and the number of people actually present in a picture was either 0,1 , 2 , or 3 . In Part III, the 15 pictures were again presented to $S$, at the same rate as in Part $I$, and $S$ stated the number of people present in each picture. In Part IV, S was given $2 \mathrm{~min}$ in which to attempt to recall the names of all 15 pictures in any order. The instructions for each part were given immediately before the part was run, the interval between successive parts being approximately 1 min. Different random presentation orders were used for Parts I, II, and III, but for a given part every S was exposed to the same order.

The 15 pictures were selected from an initial set of 493 , which had been culled from popular magazines. Fifteen judges had categorized each of these 493 pictures (or some subset of them) as pleasant $(P)$, unpleasant $(U)$, or neutral $(N)$ and the pictures finally selected included the five most extreme members of each of these categories.
The GSR was obtained from a Fels Model 22A Dermohmeter whose output was recorded on one channel of a 6-channel Offner Type $R$ polygraph. The active GSR electrode was a $21 \mathrm{~mm}$ diameter zinc disc mounted in a lucite cup and attached to the left palm, while a $40 \mathrm{~mm}$ diameter silver mesh electrode attached to the back of the left arm just above the wrist served as the inactive electrode. The slides were projected via a Kodak Carousel slide projector onto a screen which was approximately $5 \mathrm{ft}$ in front of S.

\section{Results and Discussion}

The relation between the amplitude of an individual S's GSR to a given picture during Part I and the amplitude of his GSR when he correctly recalls the number of people in that picture in Part II provides information bearing on the first question raised in the introduction. ${ }^{2}$ For each S, GSRs to the pictures in Part I and GSRs to correct recalls of these pictures in Part II were ranked on the basis of amplitude, separate rankings being done for the two parts. The resulting 12 rank order correlations between Parts I and II are presented in Table $1 .^{2}$ Only one of these correlations is significant at the .05 level, and it is negative.

Of the remaining 11 correlations, eight are positive and three negative. The average rank order correlation $(\bar{\rho})$ was only .055 and the $\mathrm{z}$ score for this value of $\bar{\rho}^{\prime}$ computed by the procedure given in Taylor \& Fong (1963), is .66, p > .05. Thus it appears that there was no consistent relation between presentation and recall GSRs. Two possible reasons for this negative finding deserve mention. First, the pictures proved to be relatively weak with regard to consistently eliciting differential GSRs. In an analysis of variance carried out on transformed GSR scores (i.e., log conductance change scores), type of picture ( $\mathrm{P}, \mathrm{U}$, or $\mathrm{N})$ did not produce a significant effect, $F(2,22)=1.21, p>.05$, but did significantly interact with Part number (I, II or III), F(2,22) $=3.02, p<.05$; unpleasant pictures were associated with the largest conductance changes in Parts I and II, and with the smallest conductance change in Part III. Second, although we considered only GSRs associated with correct responses in Part II, it is possible that these GSRs reflected differential difficulty of answering the questions.

With regard to the second question, raised in the introduction for each $S$ we determined whether the observed amount of clustering (i.e., the observed number of within-category repetitions) in Part IV exceeded that expected by a chance formulation (see Bousfield \& Putt, 1964, p. 78). Summing across the three categories ( $P, U$, and $N)$, observed clustering exceeded that expected by chance for all 12 Ss. Summing across Ss, the actual amount of clustering was greatest for $\mathrm{P}$, next for $\mathrm{U}$, and least for $\mathrm{N}$. This ordering of categories also obtained for the total number

Table 1

Rank Order Correlations $(\rho)$ between Parts I and II

\begin{tabular}{cl} 
S No. & $\rho$ \\
\hline 1 & .08 \\
2 & .20 \\
3 & $.59^{*}$ \\
4 & .13 \\
5 & .06 \\
6 & .12 \\
7 & .37 \\
8 & .46 \\
9 & .15 \\
10 & .34 \\
11 & .37 \\
12 & .17 \\
\hline
\end{tabular}

${ }^{*} p<.0 .5$ 
of items recalled, the percentages of correct recall being 97, 93, and 90 for the $P, U$, and $N$ categories, respectively.

It is possible that this clustering effect was mediated by associative clustering if $S s$ often gave associatively related names to pictures in the same category. In order to test this possibility 12 additional Ss ( 6 males and 6 females) were run in the experiment with one major modification. In Part $\mathrm{I}$, instead of $\mathrm{S}$ supplying the names, E gave $S$ names for the pictures. These names were selected so that the names for pictures in a given category bore no associative relation to one another, as determined by the recently collected Rosenberg \& Cohen (1966) norms. The free recall results were: for 2 Ss observed clustering equalled that expected by chance, while for 7 of the remaining $10 \mathrm{Ss}$ observed clustering exceeded expected clustering ( $p>.05$, by a sign test). Apparently clustering by subjective categories ( $P, U$, and $N$ ) is partially mediated by $\mathrm{Ss}^{\prime}$ naming subjectively similar experiences with associatively related names.

\section{REFERENCES}

BOUSFIELD, W. A. The occurrence of clustering in the recall of randomly arranged associates. J. gen. Psychol, 1953, 49, 229-240.
BOUSFIELD, W. A., \& PUFF, C. R. Clustering as a function of response dominance. J. exp. Psychol, 1964, 67, 76-79.

KELLER, M. Mediated generalization: The generalization of a conditioned galvanic skin response established to a pictured object. Amer. J. Psychol., $1943,56,438-448$.

ROSENBERG, S., \& COHEN, B. D. Referential processes of speakers and listeners. Psychol Rev., 1966, 73, 208-231.

TAYLOR, W. L., \& FONG, C. Some contributions to average rank correlation methods and to the distribution of the average rank correlation coefficient. J. Amer. Statist. Assn., 1963, 58, 756-769.

\section{NOTES}

1. It might be argued that the GSRs obtained in Part II reflect semantic generalization of the GSR from the picture to its name, rather than a GSR to the recall of the picture. However, Keller (1943) has shown that there is no significant generalization of GSRs from pictures to their names. Also, it might be argued that any relation between GSRs obtained in Parts I and II is due to the fact that the names were present in both parts. However, in Part I the names were not stated until after the picture was removed, and the GSRs recorded in Part I occurred mainly during picture presentation.

2 . The $n$ for each rank order correlation was the number of correctly answered questions in Part II, this number ranging from 13 to 15 . 